\title{
Caracterización integral del derrame pleural en un hospital de referencia del sureste de México
}

\author{
Comprehensive description of pleural effusion in a \\ reference center in the southeast of Mexico
}

\author{
Armando David Villarreal-Vidal, ${ }^{*}$ Gary Vargas-Mendoza, ${ }^{*}$ Arturo Cortes-Telles*
}

*Hospital Regional de Alta Especialidad de la Península de Yucatán, México.

\begin{abstract}
RESUMEN. Introducción: La etiología del derrame pleural puede presentar variaciones según el ámbito geográfico, temporal o ubicación. En México, la información disponible del derrame pleural deriva de centros hospitalarios especializados en la atención de padecimientos respiratorios. Posiblemente los resultados no reflejen la frecuencia de atención de dicho padecimiento en otros escenarios, por ejemplo, en hospitales generales. Objetivo: Conocer la etiología, manifestaciones clínicas ydiferencias del líquido pleural entre las diversas etiologías del derrame pleural en un hospital general de referencia del Sureste de México. Material y métodos: Estudio observacional, prospectivo que incluye la totalidad de casos con derrame pleural ingresados en un período de tres años. $\mathrm{Al}$ ingreso en el servicio de admisión continua se realizó toracocentesis diagnóstica y se obtuvo un historial clínico a detalle. Resultados: Se incluyeron 178 casos que ingresaron para abordaje del derrame pleural. La etiología más común fue paraneumónica (34\%). Entre los síntomas, la disnea fue el más frecuente (91\%) y no fue diferente entre las etiologías del derrame pleural $(p=0.095)$. Entre los síntomas, manifestaciones sistémicas como hiporexia, pérdida de peso se observaron con mayor frecuencia en los casos asociados a neoplasia y tuberculosis; en tanto, fiebre, manifestaciones bronquiales (tos, expectoración) y dolor torácico fueron más frecuentes en los casos de etiología infecciosa (paraneumónica y tuberculosis). Conclusiones: La etiología paraneumónica fue la causa más frecuente del derrame pleural en un hospital regional. La disnea fue el síntoma más prevalente sin diferencias entre etiologías. Los datos contrastan con lo previamente publicado, es posible que estas diferencias radiquen en el sistema de atención de los hospitales.
\end{abstract}

Palabras clave: Epidemiología, etiología, derrame pleural, toracocentesis, paraneumónico, cáncer.

\begin{abstract}
Introduction: The etiology of pleural effusion may vary depending on the geographic location or temporality. In Mexico, the information available regarding pleural effusion derives from thirdlevel reference centers specialized in respiratory diseases. Possibly the results do not reflect the prevalence of this condition in other scenarios, such as, general wards. Objective: To determine the etiology, clinical manifestations and differences of the pleural fluid analysis between several etiologies of pleural effusion in a general reference center in the southeast of Mexico. Material and methods: An observational, prospective study that includes all consecutive cases with pleural effusion admitted in a period of 3 years. On admission, a diagnostic thoracentesis was performed and detailed clinical history was obtained. Results: We included 178 cases that were admitted with pleural effusion. The leading cause was parapneumonic (34\%). Among symptoms, dyspnea was the most frequent (91\%) and was not different among several pleural effusion etiologies $(p=0.095)$. Among symptoms, systemic manifestations such as hyporexia, weight loss were more prevalent in malignant pleuraleffusions and tuberculosis; meanwhile, fever, bronchial manifestations (cough, expectoration) and chest pain were more frequent in cases of infectious etiology (parapneumonic and tuberculosis). Conclusions: Parapneumonic effusions was the most prevalent cause of pleural effusion in a general reference hospital. Dyspnea was the main symptom among several etiologies without statistical difference. The data contrast with previously published reports, possibly due to differences in the hospital's care system.
\end{abstract}

Keywords: Epidemiology, etiology, pleural effusion, thoracentesis, parapneumonic, cancer.

\section{Correspondencia:}

\section{Dr. Arturo Cortes-Telles}

Hospital Regional de Alta Especialidad de la Península de Yucatán.

México.

Correo electrónico: dr_morenheim@hotmail.com

Trabajo recibido: 02-I-2019; aceptado: 12-III-2019.

\section{INTRODUCCIÓN}

El derrame pleural (DP) se forma cuando existe un desequilibrio entre los mecanismos de producción/absorción habitual del líquido en el espacio pleural. ${ }^{1}$ Existen diversas enfermedades que pueden cursar con DP; en adultos, las más comunes incluyen: insuficiencia cardíaca, enfermeda- 
des oncológicas, secundarios a padecimientos infecciosos incluyendo neumonía o tuberculosis, así como también, padecimientos vasculares, v.gr., embolismo pulmonar agudo. ${ }^{2}$

La historia clínica y el examen físico son fundamentales durante la evaluación inicial. Los signos y síntomas varían dependiendo de la enfermedad subyacente; no obstante, la presencia de disnea, tos y dolor torácico de tipo pleurítico son comunes con intensidad variable. ${ }^{3}$

El abordaje inicial del DP incluye una radiografía posteroanterior del tórax. Con base en la extensión del DP puede realizarse una toracentesis para obtener líquido cuyo análisis permite diferenciar el escenario clínico entre trasudados o exudados acorde con los criterios vigentes. ${ }^{4-6}$ En particular, es indispensable complementar la evaluación de un exudado con los siguientes análisis en el líquido pleural: a) conteo y diferencial celular, b) $\mathrm{pH}$, c) nivel de glucosa, d) adenosin- deaminasa (ADA), e) cultivos microbiológicos y f) análisis citológico. La integración de los resultados permitará acortar el diagnóstico diferencial e iniciar un tratamiento oportuno.?

En Estados Unidos se estima que 1,5 millones de personas desarrollan DP cada año; de éstos, la principal causa es por insuficiencia cardíaca. ${ }^{8}$ Por otro lado, en España, el análisis de más de 3,000 toracocentesis permitió identificar que la principal causa de DP era la relacionada con derrames paraneoplásicos. ${ }^{9}$

En México, la información relacionada con la frecuencia de DP deriva de centros hospitalarios que atienden la mayoría de las veces padecimientos respiratorios. ${ }^{10,11}$ Los reportes son consistentes y el derrame paraneoplásico es la etiología del DP que ocupa la primera causa de atención. Si bien, la información es confiable, existe la posibilidad de que no refleje la situación en escenarios hospitalarios donde existen salas generales de medicina interna como el principal servicio de atención. La falta de información que permita seleccionar e interpretar adecuadamente las pruebas empleadas en el abordaje diagnóstico del DP acorde con el escenario clínico más probable, puede condicionar errores o retrasos en el diagnóstico y tratamiento.

Por lo tanto, el objetivo de nuestro estudio es conocer la frecuencia y etiología del DP; asimismo, contrastar las manifestaciones clínicas y del líquido pleural entre las diversas etiologías en un hospital de referencia general del sureste de México.

\section{MATERIAL Y MÉTODOS}

\section{Pacientes}

Estudio observacional, transversal, prospectivo que incluyó pacientes mayores de 18 años con diagnóstico de DP en estudio que ingresaron de forma consecutiva al Hospital Regional de Alta Especialidad de la Península de Yucatán
(HRAEPY) del $1^{\circ}$ de enero del 2015 al 31 de diciembre del 2017. Se excluyeron los pacientes con diagnóstico de DP y abordaje previo incompleto y los casos con información clínica o paraclínica insuficiente para definir la etiología o las características del DP. El estudio fue aprobado por los Comités de Investigación y Ética en Investigación del hospital con el número 2015-011. Más aún, todos los datos fueron consignados en la computadora del investigador principal y se protegieron con clave siguiendo en todos momentos los estatutos vigentes de la Declaración de Helsinki. ${ }^{12}$

\section{Procedimiento}

Durante el ingreso al servicio de admisión continua y previa firma del consentimiento informado, se realizó una toracocentesis diagnóstica acorde con el procedimiento estandarizado vigente. ${ }^{3}$ En promedio se extrajeron de cada procedimiento 50 a $100 \mathrm{ml}$ de líquido pleural que fueron distribuidos para los siguientes análisis: a) citológico, b) estudio físico y citoquímico, c) cultivo y d) análisis ácido-base.

Como parte de la evaluación inicial, de forma sistematizada, se interrogó a los pacientes para obtener información médica previa (v.gr., morbilidades, tabaquismo), asimismo, los síntomas basados en la evolución del padecimiento. Los resultados del líquido pleural se extrajeron del registro electrónico brindado por el laboratorio clínico una vez que fueron reportados incluyendo: concentración de proteínas, deshidrogenasa láctica (DHL), glucosa, colesterol, triglicéridos, lactato, procalcitonina (PCT) y proteína c reactiva (PCR); también, valor del $\mathrm{pH}$, porcentaje del diferencial celular (polimorfonucleares, linfocitos, otros), reacción en cadena de la polimerasa (PCR) para Mycobacterium tuberculosis, adenosin-deaminasa (ADA); reporte de patología y cultivos con análisis de tinción de Gram, tinta china, $\mathrm{KOH}$, BAAR. A nivel sérico todos los pacientes contaron, entre otros estudios, con análisis de biometría hemática, concentración de proteínas, DHL, PCR y PCT. La diferenciación entre exudados y trasudados se realizó mediante los criterios de Light. ${ }^{6}$ En los casos con elevada sospecha de tuberculosis pleural u origen oncológico, la confirmación diagnóstica se complementó mediante biopsia pleural dirigida por videotoracoscopia.

Por otro lado, se revisaron las radiografías de tórax realizadas a cada paciente durante el ingreso para definir el porcentaje aproximado de ocupación del DP en el hemitórax afectado.

\section{Análisis estadístico}

Las variables continuas se presentan como medias con desviación estándar (DE) o medianas con intervalo intercuartílico (IIC) según sea el caso; por otro lado las variables categóricas como frecuencias con porcentajes. 
Para conocer diferencias de las variables continuas entre los grupos de estudio (etiología del derrame pleural) se llevó a cabo un análisis de varianza de una sola vía (ANOVA). El contraste intergrupos se llevó a cabo mediante un análisis post hoc con ajuste por Bonferroni.

Se consideró como estadísticamente significativo un valor de p menor a 0.05 (bimarginal). El análisis estadístico se llevó a cabo empleando el paquete STATA versión 14. (StataCorp LP, CollegeStation, Tx).

\section{RESULTADOS}

\section{Pacientes}

El presente informe incluye 178 casos que fueron documentados en el período de tiempo especificado. La edad promedio fue de 56 años ( $D E \pm 17$ ) y el $54.5 \%$ fueron hombres. Entre los antecedentes de relevancia, $37 \%$ de los pacientes tenían historia de tabaquismo con índice tabáquico de 5 paquetes/ año (RIC 5-12). Las comorbilidades más frecuentes fueron hipertensión arterial sistémica (HAS) (36\%), historia de enfermedad oncológica (24\%) y DM2 (22\%). El síntoma principal fue disnea (91\%). Por último, 34\% de los casos presentaron una opacidad por imagen que ocupaba alrededor del $50 \%$ del hemitórax comprometido. El resto de las características generales de la población se describen en la tabla 1.

\section{Etiología del derrame pleural}

La primera causa de atención por DP en nuestro hospital fue paraneumónica (34\%). Le siguieron en frecuencia los trasudados (26\%) y asociado a neoplasia (19\%). Entre los trasudados, la causa principal fue secundaria a insuficiencia cardíaca (62\%). Por otro lado, el origen primario del derrame asociado a neoplasia fue adenocarcinoma pulmonar (26\%), seguido por enfermedad metastásica $(17 \%)$ y cáncer de mama (14\%). El resto de las etiologías se describen en la tabla 2.

\section{Análisis del líquido pleural con base en la etiología}

En la tabla 3 se presentan los contrastes en parámetros del líquido pleural entre las principales etiologías. Tanto el valor de PCR como PCT fue notablemente diferente entre los grupos de estudio; los valores más altos se registraron en los casos con derrames paraneumónicos. Respecto al comportamiento ácido-base, los valores de $\mathrm{pH}$ y glucosa fueron menores en los casos paraneumónicos, del mismo modo, los valores de DHL, gradiente de DHL (LP/sérico), leucocitos totales y neutrófilos fueron mayores en los derrames paraneumónicos.

En particular, los trasudados mostraron un comportamiento ácido-base opuesto a los procesos infecciosos (pa- raneumónico o tuberculosis) con un $\mathrm{pH}$ alcalino (mediana 7.46, IIC 7.42-7.51) y glucosa dentro de rangos normales (mediana 113 mg/dl, IIC 104-152). Más aún, la diferenciación celular fue linfocitario.

Cabe destacar, la mediana del nivel de proteínas en líquido pleural fue superior en los casos confirmados con tuberculosis u origen paraneoplásico en comparación con el

Tabla 1: Características generales de la población en estudio $(n=178)$.

\begin{tabular}{|c|c|}
\hline Variables & Frecuencia (\%) \\
\hline Edad (años) & $56 \pm 17$ \\
\hline Género masculino & $97(54.5)$ \\
\hline \multicolumn{2}{|l|}{ Estado de procedencia } \\
\hline Yucatán & $144(81)$ \\
\hline Quintana Roo & $24(13.5)$ \\
\hline Campeche & $9(5)$ \\
\hline Veracruz & $1(0.5)$ \\
\hline Tabaquismo & $66(37)$ \\
\hline Índice tabáquico (paquetes/año) & $5(2-12)$ \\
\hline \multicolumn{2}{|l|}{ Comorbilidades } \\
\hline Hipertensión arterial & $58(36)$ \\
\hline Diabetes mellitus tipo 2 & $39(22)$ \\
\hline Oncológico & $42(24)$ \\
\hline Inmunológico & $6(3)$ \\
\hline \multicolumn{2}{|l|}{ Síntomas al ingreso } \\
\hline Tos & $99(56)$ \\
\hline Disnea & $162(91)$ \\
\hline Dolor torácico & $89(50)$ \\
\hline Intensidad & $7(5-8)$ \\
\hline Fiebre & $81(45)$ \\
\hline Cuantificación & $38.5 \pm 0.5$ \\
\hline Expectoración & $63(35)$ \\
\hline Hiporexia & $68(38)$ \\
\hline Pérdida de peso & $72(40)$ \\
\hline \multicolumn{2}{|l|}{ Tamaño del derrame } \\
\hline $1(1-25 \%)$ & $37(21)$ \\
\hline $2(26-50 \%)$ & $61(34)$ \\
\hline $3(51-75 \%)$ & $43(24)$ \\
\hline $4(76-100 \%)$ & $37(21)$ \\
\hline Libre & $98(55)$ \\
\hline 1 lóculo & $48(27)$ \\
\hline 20 más lóculos & 32 (18) \\
\hline
\end{tabular}


Neumol Cir Torax. 2019; 78 (3): 277-283

Tabla 2: Etiología del derrame pleural en la población analizada $(n=178)$.

\begin{tabular}{|l|c|}
\hline Variable & $\mathbf{n}(\%)$ \\
\hline Etiología general & \\
\hline Trasudados & $47(26)$ \\
\hline Infeccioso bacteriano & $60(34)$ \\
\hline Tuberculosis & $12(7)$ \\
\hline Paraneoplásicos & $35(19)$ \\
\hline Exudados no concluyentes & $24(14)$ \\
\hline Trasudados & \\
\hline Insuficiencia cardíaca congestiva & $29(62)$ \\
\hline Enfermedad renal crónica & $8(17)$ \\
\hline Hidrotórax hepático & $7(15)$ \\
\hline Pancreatitis & $1(2)$ \\
\hline Otros & $2(4)$ \\
\hline Paraneoplásicos & \\
\hline Pulmonar & $9(26)$ \\
\hline Gastrointestinal & $5(14)$ \\
\hline Mama & $5(14)$ \\
\hline Hematológico & $2(6)$ \\
\hline Primario desconocido & $3(9)$ \\
\hline Otros & $6(17)$ \\
\hline No identificables & $5(14)$ \\
\hline & \\
\hline
\end{tabular}

resto de las etiologías (5.1 g/dl y $4.3 \mathrm{~g} / \mathrm{dl}$, respectivamente); en adición, el pH inicial se encontró en rangos normales y la diferenciación celular fue con predominio linfocitario (tabla 3).

\section{Diferencias clínicas entre las etiologías del derrame pleural}

El síntoma cardinal en los casos con DP independiente de la etiología fue la disnea (91\%). En frecuencia, le siguió tos (56\%) y dolor torácico (50\%). El resto de los descriptores clínicos se describen en la tabla 1.

Al contrastar los síntomas entre los grupos de estudio, la menor frecuencia de síntomas ocurrieron en los trasudados a excepción de la disnea cuya frecuencia no fue diferente entre el resto de las etiologías $(p=0.093)$. Por otro lado, tos, dolor torácico, fiebre y expectoración fueron los síntomas más prevalentes en los derrames infecciosos tanto paraneumónicos $(72 \%, 78 \%, 88 \%$ y $62 \%$, respectivamente) como por tuberculosis $(67 \%, 58 \%, 75 \%$ y $42 \%$, respectivamente). La presencia de síntomas constitutivos como pérdida de peso se registró con mayor frecuencia en los DP por tuberculosis y asociados a neoplasia $(75 \%$ y $74 \%$, respectivamente). Finalmente, entre las morbilidades relacionadas a cada una de las etiologías, DM2 (55\%) y HAS (51\%) fueron los antecedentes más comunes en los trasudados. A su vez, la presencia de mala higiene oral fue más común en los casos con derrame paraneumónico (60\% de los casos). El resto de los contrastes clínicos se presentan en la tabla 4.

\section{DISCUSIÓN}

Con base en nuestro conocimiento, éste es el primer reporte que contrasta las características clínicas y el perfil bioquímico del líquido pleural en un grupo consecutivo de pacientes con DP atendidos en un hospital no exclusivo para enfermedades respiratorias en México. Los principales hallazgos se describen a continuación: 1) la principal causa del DP es de origen infeccioso (paraneumónico); 2) uno de cada 4 casos con DP son de tipo trasudado y la etiología más frecuente es secundario a insuficiencia cardíaca; 3) la adición sistemática de biomarcadores pleurales como PCR y PCT permite diferenciar la etiología del DP; 4) la disnea es el síntoma principal del DP independiente de la etiología y los casos con trasudados expresan la menor tasa de síntomas.

En general, cuatro de cada 10 casos atendidos en nuestra unidad fueron secundarios a padecimientos infecciosos; de éstos, $83 \%$ paraneumónico y $17 \%$ por tuberculosis. Mientras que 1 de cada 5 casos fueron paraneoplásicos. Los referentes a nivel mundial señalan a la insuficiencia cardíaca como la primera causa de atención por DP en Estados Unidos; en tanto, el registro más grande de toracocentesis diagnósticas en España reportan como primera causa de atención al derrame asociado con neoplasia., ${ }^{8,9}$ En México, los registros que derivan de centros especializados en enfermedades respiratorias son consistentes con los informes de España. ${ }^{10,11}$ De cierto, los registros son muy confiables, sin embargo, contrarios a lo observado en el presente informe. De esta manera, respaldamos nuestra hipótesis sobre la posible falta de aplicación en escenarios hospitalarios donde no se atiende exclusivamente enfermedades respiratorias; además, nuestros resultados pudieran aproximarse a lo reflejado en hospitales donde cuentan con un servicio base de medicina interna encargado de la atención de los padecimientos infecciosos, metabólicos o cardiovasculares que pueden incidir en la génesis de DP. ${ }^{13}$ Cabe destacar, que siempre se debe considerar la frecuencia relativa de las patologías acorde con la epidemiología de la región., ${ }^{9,14}$

En general, las principales etiologías de trasudados lo representan insuficiencia cardíaca, cirrosis y síndrome nefrótico; en el presente informe, 1 de cada 4 casos abordados por DP fueron trasudados y el $62 \%$ secundario a 
insuficiencia cardíaca. Proporcionalmente, esta frecuencia es mayor al compararse con el informe de más de 3,000 procedimientos de Porcel y colaboradores. ${ }^{9}$ Entre las posibles explicaciones que los autores tomamos en cuenta, está la elevada frecuencia de padecimientos cardiovasculares que son atendidos en nuestro hospital. Si bien, los trasudados son considerados como condiciones benignas, información reciente ha destacado la asociación con riesgo de muerte a 1 año. ${ }^{15}$ Casi siempre el manejo está enfocado en la optimización de la enfermedad subyacente y es común la recurrencia del DP, pero con la información disponible concluimos que los casos con refractariedad deben ser evaluados de forma multidisciplinaria para recibir la mejor estrategia terapéutica y reducir los riesgos de complicaciones subsecuentes. ${ }^{16}$ De manera interesante, en nuestra serie la segunda causa de trasudados fue la enfermedad renal crónica (ERC). Walker y colegas. ${ }^{15}$ señalan que la presencia de DP en pacientes con ERC confiere un riesgo de muerte 3 veces superior que los pacientes con ERC en tratamiento con hemodiálisis (HD) sin DP.

En relación con los derrames asociados a neoplasia encontramos que la principal causa atribuible fue cáncer pulmonar (26\%). Es conocido que la presencia de DP en pacientes con enfermedad oncológica disminuye el pronóstico de sobrevida (promedio 4-7 meses a partir del diagnóstico); en particular cuando el origen deriva del sistema respiratorio o tracto gastrointestinal..$^{17} \mathrm{El}$ reconocimiento oportuno permitirá brindar estrategias de paliación que mejoren las condiciones generales de vida (pleurodesis química o mediante videotoracoscopia, colocación de catéteres tunelizados, uso de morfina, entre otros)..$^{18}$

La cuantificación pleural de biomarcadores sistémicos como PCR y PCT contribuyen en la diferenciación de padecimientos que requieren una intervención quirúrgica temprana, v.gr., derrames paraneumónicos complicados. ${ }^{19}$ Porcel y colaboradores han informado que un valor de PCR mayor de $45 \mathrm{mg} / \mathrm{l}$ posee un «likelihood ratio» (LR) positivo de 8 para identificar un DP tipo infeccioso no complicado. ${ }^{20}$ En México, hemos informado previamente el rendimiento de PCR en suero, un valor de $143 \mathrm{mg} / \mathrm{l}$ confiere un LR positivo de 5.3 para casos con derrames paraneumónicos complicados o empiemas. ${ }^{21}$ Con base en nuestro conocimiento, es el primer informe que describe en población mexicana el rendimiento de ambos biomar-

Tabla 3: Contraste de variables en el líquido pleural con base en etiología primaria del derrame unilateral $(n=178)$.

\begin{tabular}{|c|c|c|c|c|c|c|}
\hline Variable & $\begin{array}{l}\text { Trasudados } \\
(n=47)\end{array}$ & $\begin{array}{l}\text { Paraneumónico } \\
\qquad(n=60)\end{array}$ & $\begin{array}{l}\text { Tuberculosis } \\
\qquad(n=12)\end{array}$ & $\begin{array}{l}\text { Paraneoplásico } \\
\qquad(n=35)\end{array}$ & $\begin{array}{l}\text { Otros exudados } \\
\qquad(n=24)\end{array}$ & $p$ \\
\hline Procalcitonina (ng/ml) & $0.20(0.05-0.36)$ & $1.51(1.22-1.65)$ & 0.8 & $0.1(0.1-0.16)$ & 0.05 & $0.009 \pi$ \\
\hline $\mathrm{pH}$ & $7.46(7.42-7.51)$ & $7(6.8-7.3)$ & $7.44(7.29-7.48)$ & $7.42(7.32-7.45)$ & 7.39 (7.35-7.47) & $<0.001$ \\
\hline Glucosa (mg/dl) & $113(104-152)$ & $29(2-63)$ & $88(78-95)$ & $89(52-116)$ & $110(92-130)$ & $0.005^{*}$ \\
\hline Gradiente albúmina & $2.1(1.4-2.3)$ & $0.75(0.35-1.5)$ & $0.9(0.8-1)$ & $1(0.6-1.1)$ & $1.2(1-1.5)$ & 0.101 \\
\hline Proteínas (gr/dl) & $2.32(1.64-3.05)$ & $3.55(1.98-5.18)$ & $5.1(4.5-5.5)$ & $4.3(3.8-5)$ & $4.27(3.34-5.7)$ & $0.002^{\text {भाก }}$ \\
\hline Gradiente proteínas LP/S & $0.34(0.26-0.5)$ & $0.77(0.65-0.86)$ & $0.79(0.65-1.13)$ & $0.68(0.62-0.80)$ & $0.67(0.52-0.74)$ & 0.115 \\
\hline $\mathrm{DHL}(\mathrm{UI} / \mathrm{I})$ & $145(133-200)$ & $2,244(1,170-6,703)$ & $616(271-903)$ & $690(346-1109)$ & $500(244-885)$ & $<0.001^{\S \S}$ \\
\hline Neutrófilos (\%) & $23(20-39)$ & $86(70-92)$ & $20(9-55)$ & $15(6-25)$ & $20(10-24)$ & $<0.001$ \\
\hline Linfocitos (\%) & $73(61-80)$ & $20(8-30)$ & $79(44-90)$ & $86(75-95)$ & $81(76-90)$ & $<0.001$ \\
\hline $\mathrm{ADA}(\mathrm{UI} / \mathrm{l})$ & $5.1(2.1-7.2)$ & $15(11.4-18)$ & $9.8(6.2-23.6)$ & $5(2-8.7)$ & $10.9(5.4-18)$ & $<0.001 \|$ \\
\hline Colesterol (mg/dl) & $23(20-41)$ & $\mathrm{N} / \mathrm{D}$ & 136 & $67(65-102)$ & $79(30-133)$ & 0.109 \\
\hline Triglicéridos (mg/dl) & $30(23-64)$ & $46(25-74)$ & $49(41-52)$ & $41(30-55)$ & $54(31-114)$ & $0.028^{* *}$ \\
\hline
\end{tabular}

Nota: Todas las variables fueron obtenidas del líquido pleural; asimismo, los valores se presentan como medianas con intervalo intercuartílico. N/D = No disponible. PCT = Procalcitonina. $\mathrm{PCR}=$ Proteína $\mathrm{c}$ reactiva. $\mathrm{LP} / \mathrm{S}=$ Líquido pleural/sérico. $\mathrm{ADA}=$ Adenosin-deaminasa.

ANOVA con ajuste post hoc por Bonferroni: "Paraneumónico versus paraneoplásico, $p=0.012$. ${ }^{\circledR}$ Paraneumónicos versus trasudados o para neoplásicos, $p<0.04$. ${ }^{\circledR}$ araneumónico versus otros grupos $p<0.01$. "Tuberculosis versus paraneumónico, $p=0.015$. "TiTuberculosis o paraneoplásico u otros exudados versus trasudado, $p<0.03$. ${ }^{\S \S} P$ araneumónico versus otros, $p<0.005$."Paraneumónico versus trasudado o paraneoplásico, $p<0.01$. **otros exudados versus paraneumónicos, $p<0.03$. 
Tabla 4: Caracterización clínica y morbilidades entre las etiologías del derrame pleural $(n=178)$.

\begin{tabular}{|c|c|c|c|c|c|c|}
\hline Variables & $\begin{array}{l}\text { Trasudados } \\
\quad(n=47)\end{array}$ & $\begin{array}{l}\text { Paraneumónico } \\
(n=60)\end{array}$ & $\begin{array}{l}\text { Tuberculosis } \\
\qquad(n=12)\end{array}$ & $\begin{array}{l}\text { Paraneoplásico } \\
\quad(n=35)\end{array}$ & $\begin{array}{l}\text { Otros exudados } \\
\qquad(n=24)\end{array}$ & $p$ \\
\hline Edad (años) & $67(51-78)$ & $54(41-65)$ & $53(41-59)$ & $59(42-68)$ & $57(34-69)$ & 0.002 \\
\hline \multicolumn{7}{|l|}{ Síntomas } \\
\hline Tos & $18(38 \%)$ & $43(72 \%)$ & $8(67 \%)$ & $17(49 \%)$ & $13(54 \%)$ & 0.010 \\
\hline Disnea & $45(96 \%)$ & $54(90 \%)$ & $10(83 \%)$ & $34(97 \%)$ & $19(80 \%)$ & 0.095 \\
\hline Dolor torácico & $8(17 \%)$ & $47(78 \%)$ & $7(58 \%)$ & $16(46 \%)$ & $10(42 \%)$ & $<0.001$ \\
\hline Intensidad (EVA) & $6.5(5-7.5)$ & $7(5-8)$ & $5(4-8)$ & $7(6.5-8.5)$ & $7(6-7)$ & 0.101 \\
\hline Fiebre & $3(6 \%)$ & $53(88 \%)$ & $9(75 \%)$ & $3(9 \%)$ & $9(37 \%)$ & $<0.001$ \\
\hline Cuantificación $\left({ }^{\circ} \mathrm{C}\right)$ & $38.2(38-38.5)$ & $38.5(38-38.5)$ & $38.5(38.2-38.8)$ & $39(38-39)$ & $39(38.5-39)$ & 0.983 \\
\hline Expectoración & $8(17 \%)$ & $37(62 \%)$ & $5(42 \%)$ & $5(14 \%)$ & $8(33 \%)$ & $<0.001$ \\
\hline Hiporexia & $9(19 \%)$ & $28(47 \%)$ & $6(50 \%)$ & $16(46 \%)$ & $9(37 \%)$ & 0.031 \\
\hline Pérdida de peso & $3(6 \%)$ & $23(38 \%)$ & $9(75 \%)$ & $26(74 \%)$ & $11(46 \%)$ & $<0.001$ \\
\hline \multicolumn{7}{|l|}{ Morbilidades } \\
\hline Tabaquismo & $12(25 \%)$ & $25(42 \%)$ & $6(50 \%)$ & $11(31 \%)$ & $12(50 \%)$ & 0.179 \\
\hline IT (paquetes/año) & $11(2.2-31.8)$ & $5(2-10.5)$ & $3.9(2-7)$ & $6.9(1.7-19.3)$ & $5(1.2-11.4)$ & 0.121 \\
\hline HAS & $24(51 \%)$ & $25(42 \%)$ & $1(8 \%)$ & $3(9 \%)$ & $5(21 \%)$ & $<0.001$ \\
\hline DM2 & $26(55 \%)$ & $2(3 \%)$ & $1(8 \%)$ & $7(20 \%)$ & $3(12 \%)$ & $<0.001$ \\
\hline Enfermedad oncológica & $1(2 \%)$ & $5(8 \%)$ & $0(0 \%)$ & $28(80 \%)$ & $8(33 \%)$ & $<0.001$ \\
\hline $\begin{array}{l}\text { Enfermedad } \\
\text { inmunológica }\end{array}$ & $2(4 \%)$ & $1(2 \%)$ & $0(0 \%)$ & $0(0 \%)$ & $3(12 \%)$ & 0.124 \\
\hline Mala higiene oral & $9(19 \%)$ & $36(60 \%)$ & $4(33 \%)$ & $4(11 \%)$ & $9(37 \%)$ & $<0.001$ \\
\hline
\end{tabular}

Nota $=$ Los valores se presentan como medianas con intervalo intercuartílico o frecuencias con porcentajes.

$\mathrm{EVA}=$ Escala visual análoga. IT = Índice tabáquico.

cadores cuantificados entre los diferentes padecimientos pleurales. Nuestros hallazgos muestran los contrastes de los biomarcadores entre las diferentes patologías y fue evidente que los derrames paraneumónicos tuvieron los valores más altos al compararse con las otras patologías pleurales. Cabe recordar que los pacientes con infecciones del espacio pleural tienen el potencial para deterioro clínico dado el estado inflamatorio subyacente y en casos con empiema deben ser drenados lo antes posible. ${ }^{22}$

Acorde con la expresión de los síntomas, la disnea es la manifestación principal en los padecimientos pleurales independiente de la causa. Más aún, la caracterización integral de los síntomas nos permitió evidenciar mayor frecuencia de compromiso sistémico (hiporexia y pérdida de peso) en padecimientos de etiología oncológica o tuberculosis, por otro lado, las manifestaciones bronquiales fueron las predominantes en padecimientos de causa infecciosa (paraneumónicos y tuberculosis). De manera interesante, los trasudados tienen la menor frecuencia de síntomas en general, con excepción de la disnea.
Finalmente, los casos con trasudados tuvieron una mayor frecuencia de morbilidades como DM2 e hipertensión arterial sistémica. Este hallazgo es esperado, dado que ambas entidades clínicas son los factores de riesgo más sólidos para el desarrollo subsecuente de insuficiencia cardíaca y ERC. ${ }^{23}$ Además, la existencia de mala higiene oral tuvo una mayor frecuencia de asociación con los derrames infecciosos (paraneumónico y tuberculosis). Este dato debe ser considerado como un factor de riesgo, tanto para el desarrollo de DP como para la complicación del padecimiento que amerite resolución vía quirúrgica. ${ }^{24}$

Entre las limitantes de nuestro estudio podemos señalar el origen unicéntrico de los resultados, empero, es el primer informe en México que describe integralmente a la patología pleural durante la evaluación inicial. Del mismo modo, el estudio incluye los casos que fueron referidos para abordaje del DP, existe la posibilidad de no haber incluido casos poco representados clínicamente donde el médico tratante asume que la causa del DP es por la enfermedad subyacente (principalmente insuficiencia cardíaca, 
enfermedad renal crónica). La cuantificación del ADA fue mediante el método enzimático colorimétrico, no obstante, todos los casos con sospecha de tuberculosis pleural fueron intervenidos para biopsia pleural vía toracoscopia.

\section{CONCLUSIONES}

En el presente informe la principal causa de atención por DP fueron derrames paraneumónicos, trasudados y padecimientos oncológicos, en orden consecutivo. La disnea fue la manifestación clínica inicial del DP independientemente de la etiología. Estos resultados pueden considerarse para ser aplicados en escenarios hospitalarios donde no se atiende de manera exclusiva enfermedades respiratorias, v.gr., hospitales generales.

\section{REFERENCIAS}

1. Feller-Kopman D, Light R. Pleural disease. N Engl J Med 2018;378(8):740-751. doi: 10.1056/NEJMra1403503.

2. Porcel JM, Light RW. Pleural effusions. Dis Mon 2013;59(2):29-57. doi: 10.1016/..disamonth.2012.11.002.

3. Hooper C, Lee YC, Maskell N; Group BTSPleural Guideline Group. Investigation of a unilateral pleural effusion in adults: British Thoracic Society Pleural Disease Guideline 2010. Thorax 2010;65 Suppl 2:ii417. doi: 10.1136/thx.2010.136978.

4. McGrath EE, Anderson PB. Diagnosis of pleural effusion: a systematic approach. Am J Crit Care 2011;20(2):119-127. doi: 10.4037/ ajcc2011685.

5. Light RW, Macgregor MI, Luchsinger PC, Ball WC, Jr. Pleural effusions: the diagnostic separation of transudates and exudates. Ann Intern Med 1972;77(4):507-513.

6. Light RW. The Light criteria: the beginning and why they are useful 40 years later. Clin Chest Med 2013;34(1):21-26. doi: 10.1016/j. ccm.2012.11.006.

7. Maldonado F, Lentz RJ, Light RW. Diagnostic approach to pleural diseases: new tricks for an old trade. F1000Res 2017;6:1135. doi: 10.12688/f1000research.11646.1.

8. Light RW. Pleural effusions. Med Clin North Am 2011;95(6):1055-1070. doi: 10.1016/.mcna.2011.08.005.

9. Porcel JM, Esquerda A, Vives M, Bielsa S. Etiology of pleural effusions: analysis of more than 3,000 consecutive thoracenteses. Arch Bronconeumol 2014;50(5):161-165. doi: 10.1016/..arbres.2013.11.007.

10. Sosa-Juárez A, García-Sancho C, Sánchez-Hernández JD, et al. Epidemiología del derrame pleural en el INER, 2011-2012. Neumol Cir Torax 2013;72(2):136-141.

11. Mejía-Olivares B, Valdez-López HG, Martínez-Delgado IA, et al. Etiología y prevalencia del derrame pleural en la UMAE No. 34 Monterrey, Nuevo León, México.Neumol Cir Torax 2013;72(1):20-24.
12. Riis P. Thirty years of bioethics: the Helsinki Declaration 1964-2003. New Rev Bioeth 2003;1(1):15-25.

13. Bhatnagar R, Maskell NA. Treatment of complicated pleural effusions in 2013. Clin Chest Med 2013;34(1):47-62. doi: 10.1016/j. ccm.2012.11.004.

14. Villena V, López Encuentra A, Echave-Sustaeta J, Alvarez Martínez C, Mariin Escribano P. [Prospective study of 1,000 consecutive patients with pleural effusion. Etiology of the effusion and characteristics of the patients]. Arch Bronconeumol 2002;38(1):21-26.

15. Walker SP, Morley AJ, Stadon L, et al. Nonmalignant pleural effusions: a prospective study of 356 consecutive unselected patients. Chest 2017;151(5):1099-1105.doi: 10.1016/j.chest.2016.12.014.

16. Bintcliffe OJ, Lee GY, Rahman NM, Maskell NA. The management of benign non-infective pleural effusions. Eur Respir Rev 2016;25(141):303-316. doi: 10.1183/16000617.0026-2016.

17. Porcel JM, Gasol A, Bielsa S, Civit C, Light RW, Salud A. Clinical features and survival of lung cancer patients with pleural effusions. Respirology 2015;20(4):654-659.doi: 10.1111/resp.12496.

18. Bibby AC, Dorn P, Psallidas I, et al. ERS/EACTS statement on the management of malignant pleural effusions. Eur Respir J 2018;52(1): pii: 1800349. doi: 10.1183/13993003.00349-2018.

19. Bielsa S, Valencia H, Ruiz-González A, Esquerda A, Porcel JM. Serum C-reactive protein as an adjunct for identifying complicated parapneumonic effusions. Lung 2014;192(4):577-581.doi: 10.1007/ s00408-014-9606-5.

20. Porcel JM, Bielsa S, Esquerda A, Ruiz-González A, Falguera M. Pleural fluid C-reactive protein contributes to the diagnosis and assessment of severity of parapneumonic effusions. Eur J Intern Med 2012;23(5):447-450. doi: 10.1016/j.ejim.2012.03.002.

21. Vega-Sánchez AE, Che-Morales JL, Vargas-Mendoza GK, ManjarrezMartín DA, Cortés-Telles A. Procalcitonina sérica como biomarcador diagnóstico de derrame paraneumónico o empiema. Neumol Cir Torax 2017;76(1):7-13.

22. Sahn SA, Light RW. The sun should never set on a parapneumonic effusion. Chest 1989;95(5):945-947.

23. Stevens PE, Levin A; Kidney Disease: Improving Global Outcomes Chronic Kidney Disease Guideline Development Work Group Members. Evaluation and management of chronic kidney disease: synopsis of the kidney disease: improving global outcomes 2012 clinical practice guideline. Ann Intern Med 2013;158(11):825-830.doi: 10.7326/0003-4819-158-11-201306040-00007.

24. Davies HE, Davies RJ, Davies CW; Group BTSPleural Disease Guideline Group. Management of pleural infection in adults: British Thoracic Society Pleural Disease Guideline 2010. Thorax 2010;65 Suppl 2:ii41-53. doi: 10.1136/thx.2010.137000.

Conflicto de intereses: Los autores declaran no tener conflicto de intereses. 